\title{
Síndrome de Rokitansky - Kuster - Hauser
}

Dra. Maria Inés Sims D. ${ }^{1}$; Dra. Margarita Reyes R. ${ }^{2}$; Dr. Alberto Costoya A. ${ }^{3}$

\section{Rokitansky - Kuster - Hauser Syndrome}

\begin{abstract}
Two clinical cases of Rakitansky-Küster-Hauser syndrome are shown. The first case was a seventeen years old girl carrier of a congenital heart malformation already operated on, who was consulting because of a primary amenorhea. The second one was a toun year old pacient, in wicls clinical examination because of vulvoraginitis, leaded to the diagnosis.

The most uscfull me thods for diagnosis are commented and also the application of new technics.
\end{abstract}

Entre las anomalías úterovaginales congénitas se encuentra el sindrome de Rokitansky-KüsterHauser, el cual es muy poco frecuente y su diagnóstico se hace principalmente en la edad adulta. Con el objeto de difundir entre los pediatras este sindrome, se presentan dos casos estudiados en la Unidad de Ginecologia Infantil y Adolescencia de nuestro Hospital.

La "aplasia Mülleriana" se caracteriza por ausencia de los derivados Müllerianos: trompas,

1 Jefe Unidad Ginecología Infantil y Adolescencia. Hospital Luis Calvo Mackenna.

${ }^{2}$ Unidad Ginecología Infantil y Adolescencia. Hospital Luis Calvo Mackenna.

3 Departamento Ginecología y Obstetricia. Hospitaf Militar. cuerpo uterino, cuello y porción alta de la vagina.

Ocasionalmente persisten remanentes fibromusculares o trompas de Falopio rudimentarjas. Los genjtales externos son normales, el himen es normal y la vagina termina ciega.

Durante la adolescencia se desarrollan los caracteres sexuales secundarios en forma normal, incluyendo desarrollo mamario, vello pubiano y axilar y un apropiado incremento en el tamaño de los genitales externos.

Los ovarios son normales. Los niveles de estrógenos y gonadotrofinas también son normales.

En cuanto a la etiologia, los estudios citogenéti. cos son normales y no se han demostrado factores teratogénicos.

Suele asociarse con malformaciones del tracto urogenital y esqueléticas (vertebrales). 


\section{Caso 1.}

A.I.P.R. Consultó a los 17 años 10 meses por amenorrea primariz. A los 5 años fue operada de Tetralogía de Fallot. Telarquia a los 15 años. Pubarquia y vello axilar a los 15 años y medio. Padres y henmanos sanos.

Estado nutricional normal, cicatrices quirírgicas de toracotomías. Mamas normales con desarroflo de tipo III; vello axilar presente. Examen abdominal normal. Vello pubiano con desarrollo tipo III, escaso desarrollo de las ninfas; introito vaginal rosado, himen delgado. En el tracto rectal con palpación bimanual no se logra palpar útero ni anexos.

LH. 1,5 M.I.U./ml; FSH 2,9 M.I.U./ml; Cromatina sexual positiva $28 \%$.

En la pelvineumografia, con $300 \mathrm{cc}$ de aire intraperitoneal, los ovarios estaban algo aumentados de tamaño; y no se veía el útero (Fig. 1 a y b).

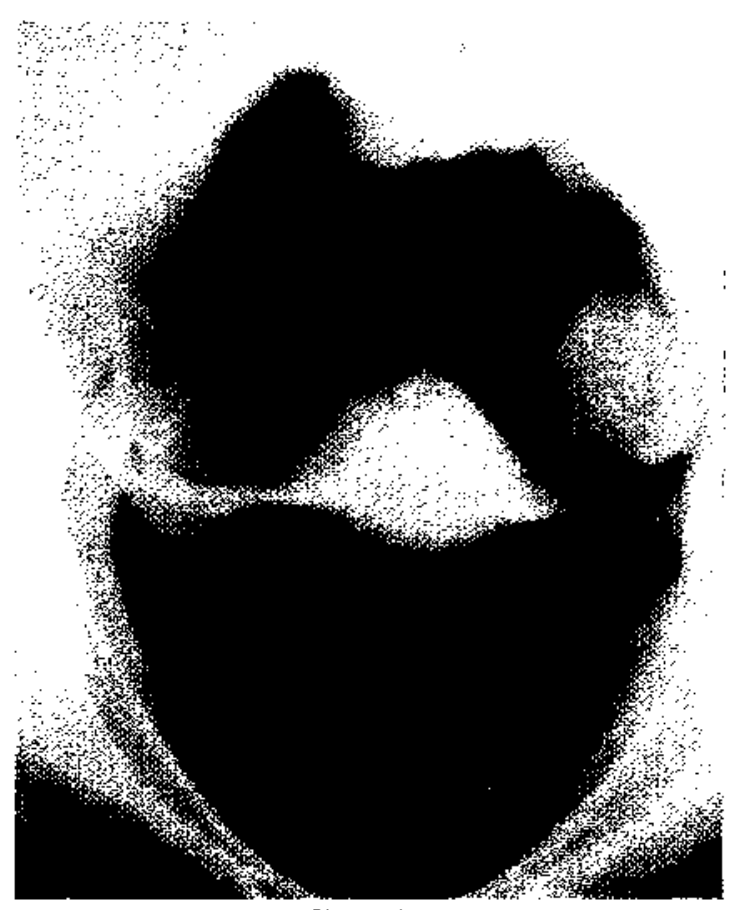

Figura 1, a

Pelvineumografía normal

Radiografías de columna, cráneo y pielografías de eliminación normales.

Laparoscopía: No se observó cuerpo uterino en la pelvis, en su lugar se encontró una delgada banda fibrosa (Fig. 2). Ambas trompas y ovarios eran de aspecto normal.

En la biopsia ovárica bilateral obtenida a través del laparoscopio (Fig. 3) demostró que ambos ovarios tenían un número de folículos muy escasos en comparación con lo normal.

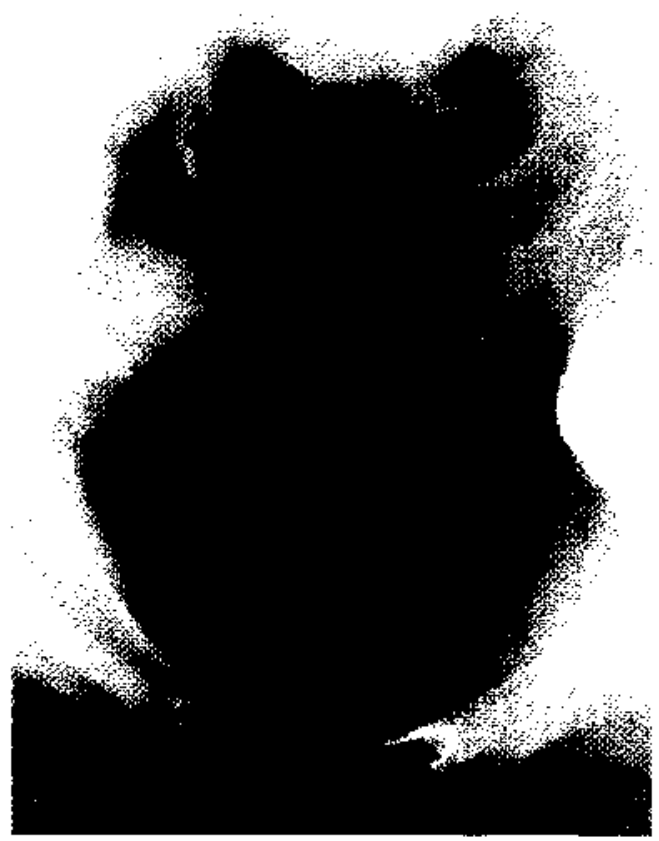

Figura 1. b

Pelvineumografía de la paciente mostrado la ausencia de utero.

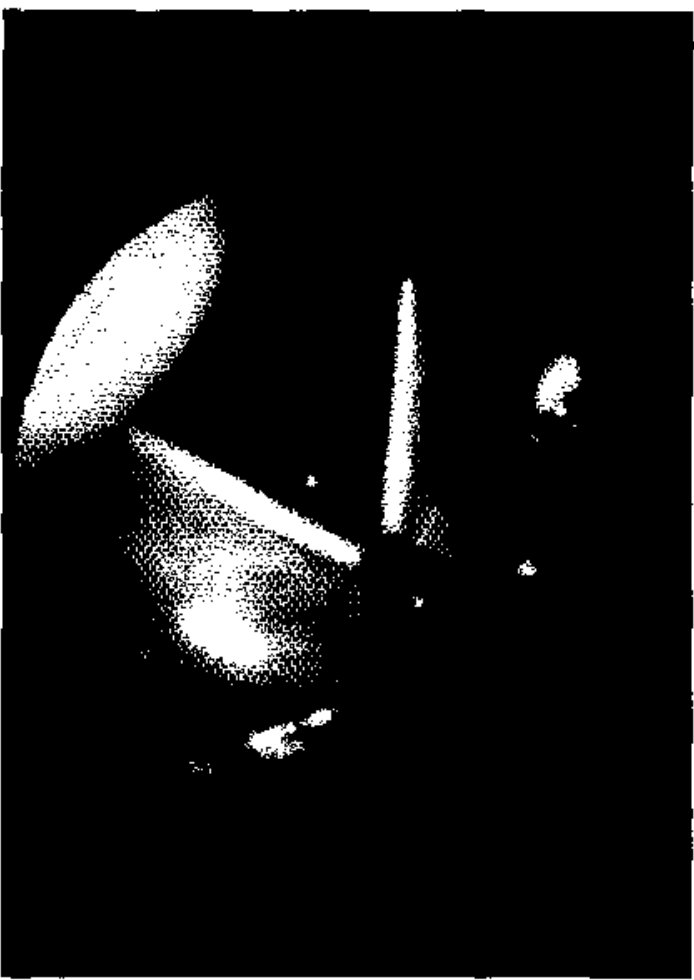

Figura 2.

Laparoscopía: ausencia de útero. En su lugar se observa una bandeleta fibrosa. 


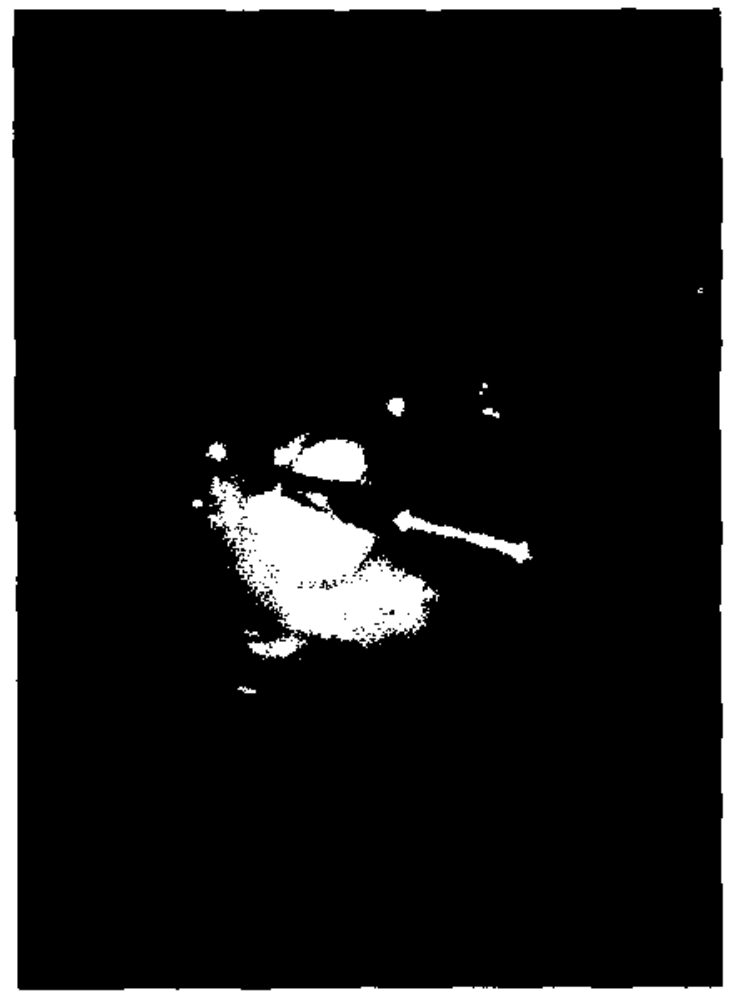

Figum 3.

Laparoscopía: Toma de biopsia orárica.

\section{Caso 2.}

M.S.V. Consultó a los 4 años 4 meses por leucorrea.

A los 8 meses sufrió desnutrición grado III, en el periodo de lactante tuvo coqueluche, otitis media y bronconeumonia. A los 4 años fue operada por estrabismo. Se ignoran sus antecedentes familiares. Su estado nutritivo era normal; mamas de tipo 1, aréolas normales. Vello axilar y pubiano ausentes. Vulva cerrada, infantili ninfas pequeñas, introito vaginal estrecho, el orificio himenal se observaba con dificultad. En el tacto rectal no se palpó nódulo uterino.

En la ecografía se veja una vagina en posición normal terminada en fondo de saco, no se encontró útero. Sus ovarios eran de aspecto normal (Fig. 4).

La columna vertebral mostraba en la radiografía leve escoleosis con vértebras normales. Uretrocistografia y pielografía de eliminación normales.

\section{COMENTARIO}

El diagnóstico de sindrome de RokitanskyKüster-Hauser en la literatura se presenta en la mayoría de los casos, como amenorrea Primaria. Hemos visto en el 2do. de nuestros casos, cómo el

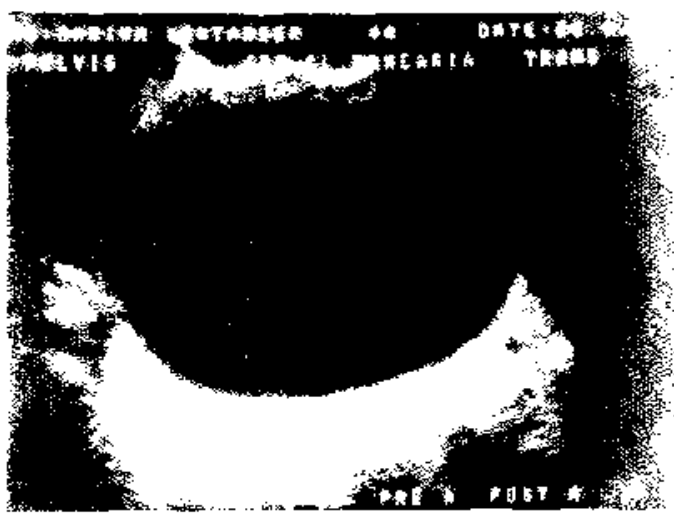

trigura 4.

Lcografia: Ausencia de útero; ovarios normales muy lateralizados.

diagnóstico puede plantearse también en etapas más precoces de la vida, en pacientes netamente pediátricos.

El estudio dirigido en esta embriopatía lleva directamente al empleo de técnicas como la ecografía. Ella es de gran ayuda, sobre todo por ser un método absolutamente incruento, no invasivo.

Nos interesa destacar la importancia en este estudio de otra técnica como la "Laparoscopia" medio de diagnóstico de gran rendimiento, pues permite en forma rápida y generalmente ambulatoria, una excelente visualización de la cavidad pelviana y abdominal. Es de destacar las posibiliđades operatorias a través de este método. Una de ellas, la posibilidad de toma de biopsia, la cual en este caso permitió corroborar la existencia de ovarios histológicamente sanos.

De todos los elementos utilizados en estos casos, el de mayor precision y certeza es, a nuestro juicio, la Laporoscopia, por las razones antes mencionadas. Este método sólo tiene la desventaja de ser "invasivo", pero las complicaciones son casi inexistentes cuando un cirujano respeta las contra. indicaciones gencrales y las locales (antecedentes de Laparotomias iterativas).

El tratamiento en el largo plazo de estas pacientes consiste en construir una neovagina mediante una plastia que se hará de acuerdo al grado de compromiso vaginal. Se aconseja realizar este tratamiento en edad adulta, en relación al inicio de una vida sexual activa.

El interés de presentar estos dos casos ha sido el de liamar la atención de los Pediatras Generales sobre la existencia de esta embriopatía. Se demuestra que a través de un simple Tacto Rectal practicado por manos especializadas, se puede sospechar el sindrome, evitando una serie de exámenes inadecuados y costosos. En cuanto a la precocidad del Diagnóstico permite preparar la paciente y a su familia sobre la plastía en el momento oportuno. 


\section{RESUMEN}

Se presentan dos casos clínicos de Síndrome de Rokitansky-Küister-Haser.

El primero, una paciente de 17 años, portadora de una cardiopatía congénita operada, que consulta por amenorrea primaria. El segundo, una paciente de 4 años, a la cual, en relación a estudio por vulvovaginitis se le practicó examen ginecológjco planteándose eldiagnóstico.

Se comenta el método de estudio y la aplica. ción de nuevas técnicas.

\section{REFERENCIAS}

${ }^{3}$ Von Muhlenbrock, $R$. et. ol.; Rokitansky-Küstcr-.
Hausers Syndrome. Rev, Chil Obt. y Ginecol. 43: 101, 1978.

2 Spas. A Spasov et. al.: Efficacy of pneumogynecography in the diagnosis of Meyer-Rokitansky-Küster- Hauser Syndrome. Am. J. Roentgenology 126: 413, 1976.

${ }^{3}$ Ravid Rosenfeld, B. Douglaslecher. Endometriosis in a parient with Rokitansky-Küster-Hauser Syndromc. American Journal of Obstetrics and Gynecology. 139: $105,1981$.

${ }_{5}^{4}$ Novak E.R. Trat. de Ginccología, 9a. Edición, Pag.'108.

${ }^{5}$ Huffman, $w$. The Gynecology of Chithood and adolesence. (Philadelphia: W.B. Saunders Company, 1968).

${ }^{6} \mathrm{John}$ M. Shane et. al.: A preliminary report on gonadotropin responsivity in the Rokitansky-KusterHauser Syndrome. American Journal of Obstetrics and Gynecology. 127: 326, 1977. 